in T acute lymphoblastic leukemias improves understanding of early human $\mathrm{T}$ lymphoid lineage commitment. Blood. 2003;101:2693-2703.

10. Roumier $\mathrm{C}$, Eclache V, Imbert M, et al. MO AML, clinical and biologic features of the disease, including AML1 genemutations: a report of 59 cases by the Groupe Francais d'Hematologie Cellulaire (GFHC) and the Groupe Francais de Cytogenetique Hematologique (GFCH). Blood. 2003;101:1277-1283.

\title{
Response
}

\section{CEBPA promoter hypermethylation in a subset of myeloid/T-lymphoid leukemias with a distinct gene expression profile}

With great interest we have read the letter by Terriou and coworkers, who have performed an extensive survey of $C E B P A$ $\mathrm{CpG}$ promoter hypermethylation in T-ALL as well as in a selection of immature acute myeloid leukemia (AML) cases. This study relates to our previous work in which we identified hypermethylation of the proximal CEBPA promoter in a small subset of AMLs with low $C E B P A$ mRNA. ${ }^{1}$ It should be noted that the patient group we described was not primarily defined by $C E B P A$ promoter hypermethylation. Instead, those cases were studied because they exhibited a unique gene expression profile. In addition to this most discriminating feature, the leukemic blasts coexpressed CD34 and T-cell antigens with myeloid markers, frequently carried NOTCH1 mutations, and expressed the transforming gene TRIB2. ${ }^{1,2}$ Using a predictive gene expression signature in an independent cohort of AML, we identified genetically and immunophenotypically similar cases with silenced CEBPA.

In the present study, Terriou et al found $C E B P A$ promoter hypermethylation in 4/54 AML cases. These 4 cases all revealed one or more T-cell characteristics (cCD3, CD7, and/or TCRG rearrangement) and may therefore resemble the leukemias we described. In T-ALL, the investigators found CEBPA promoter hypermethylation more frequently, in 37/99 cases. Importantly, however, only a minority of those T-ALLs with methylated CEBPA coexpressed myeloid surface markers (CD13 and/or CD33) and CD34. The findings of Terriou et al therefore indicate that CEBPA proximal promoter hypermethylation in combination with an immature myeloid/T-lymphoid immunophenotype is generally rare in acute leukemia, and can be found in a small percentage of AML as well as in a small fraction of T-ALL. It will be important to apply gene expression profiling to these cases to fully assess their relationship with the leukemias that we described previously.

Together, these observations highlight the challenges in classifying particular acute leukemias as AML, ALL, or a unique entity. We believe that studies as the one performed by Terriou et al will be instrumental in obtaining a better understanding of this topic. Gene expression profiling applied to these AML and T-ALL cohorts will clearly be of added value. Combining data from multiple research groups will be a requirement to address important questions regarding prognosis, and should help to evaluate according to which protocols these relatively small patient groups are best treated.

\section{Bas Wouters and Ruud Delwel}

Conflict-of-interest disclosure: The authors declare no competing financial interests.

Correspondence: Bas Wouters or Ruud Delwel, Department of Hematology, Erasmus University Medical Center, PO Box 2040, 3000CA, Rotterdam, The Netherlands; e-mail:b.wouters@erasmusmc.nl or h.delwel@erasmusmc.nl.

\section{References}

1. Wouters BJ, Jorda MA, Keeshan K, et al. Distinct gene expression profiles of acute myeloid/T-lymphoid leukemia with silenced CEBPA and mutations in NOTCH1. Blood. 2007;110:3706-3714.

2. Keeshan $\mathrm{K}, \mathrm{He} \mathrm{Y}$, Wouters BJ, et al. Tribbles homolog 2 inactivates C/EBPalpha and causes acute myelogenous leukemia. Cancer Cell. 2006;10:401-411.

\section{To the editor:}

\section{JAK2-V617F-triggered preemptive and salvage adoptive immunotherapy with donor-lymphocyte infusion in patients with myelofibrosis after allogeneic stem cell transplantation}

Primary myelofibrosis is a myeloproliferative disease, and results of conventional treatment remain unsatisfactory. ${ }^{1,2}$ Allogeneic stem cell transplantation after dose-reduced conditioning has become a reasonable, curative treatment option. ${ }^{3,4}$ Single case reports about successful donor lymphocyte infusion (DLI) for relapsed patients provided evidence of a graft-versus-myelofibrosis effect. ${ }^{5-7}$ Here, we report on 17 patients with either myelofibrosis $(n=16)$ or secondary AML post myelofibrosis $(n=1)$ and a median age of 52 years (range, 32-63 years) who received DLI from related $(\mathrm{n}=5)$ or unrelated $(\mathrm{n}=12)$ donor, either for clinical relapse (salvage DLI; $\mathrm{n}=9$ ) or residual disease monitored by JAK2 mutation level in peripheral blood (preemptive DLI; $\mathrm{n}=8$ ). Details are summarized in Table 1. One patient (no. 9) received DLI twice: once for molecular residual disease and once for reappearance of molecular disease. Sixteen patients were JAK2V617F-positive. The median time from transplantation to first DLI was 269 days (range, 127-1570 days). The median percentage of JAK2V617 mutation level in peripheral blood before first DLI was $6.2 \%$ (range, $0.2 \%-72.8 \%$ ) and significantly higher in patients with clinical relapse than with molecular relapse $(24.7 \%$ vs $0.37 \%$; $P=.03)$. The median cell dose of the first DLI was $10^{6} \mathrm{CD}^{+}$cells per kilogram of body weight (BW; range, 0.5-9 $\times 10^{6}$ cells/ $\mathrm{kg} \mathrm{BW})$. Two patients received as first DLI CD4-selected T cells $\left(5 \times 10^{6} \mathrm{CD}^{+}{ }^{+}\right.$cells $\left./ \mathrm{kg} \mathrm{BW}\right)$. Second and subsequent half-logincreased DLI were given if no graft-versus-host disease (GVHD) and no significant response were observed. The median interval between the first and the second DLI was 103 days (range, 43-661 days). The response to DLI for relapsed patients was determined by the response criteria of the International Working Group, ${ }^{8}$ and for residual disease by quantitative real time JAK2V617F polymerase chain reaction (PCR) performed from genomic DNA from peripheral blood as recently described. ${ }^{9}$ The sensitivity 